\title{
A SUBCLASS OF ANHARMONIC OSCILLATORS WHOSE EIGENFUNCTIONS HAVE NO RECURRENCE RELATIONS ${ }^{1}$
}

\author{
GARY G. GUNDERSEN
}

\begin{abstract}
The equation $w^{\prime \prime}(z)+(\lambda-p(z)) w(z)=0(z \in \mathbf{C}, \lambda \in \mathbf{R})$ with a fixed $p(z)=a_{2 m} z^{2 m}+a_{2 m-2} z^{2 m-2}+\cdots+a_{2} z^{2}\left(a_{i} \geqslant 0 \forall i, a_{2 m}>0\right)$, possesses a set of solutions $\left\{\psi_{n}(z)\right\}_{n=0}^{\infty}$ (with associated $\left\{\lambda_{n}\right\}_{n=0}^{\infty}$ ) which form a complete orthonormal set for $L^{2}(-\infty, \infty)$ (as a real space). Here it is shown that any $g(z) \psi_{N}^{(g)}(z)$ (fixed $N, q \in \mathbf{N}, g \not \equiv 0$ a polynomial with $\operatorname{deg}(g) \geqslant 1$ when $q=0$ ) cannot be expressed as a finite linear combination of $\left\{\psi_{n}(z)\right\}_{n=0}^{\infty}$, when $\operatorname{deg}(p)$ is a multiple of 4 . It is well known that $g(z) \psi_{N}^{(g)}(z)$ can always be expressed as such when $p(z)=z^{2}$ (the Hermite case).
\end{abstract}

I. Introduction. For a fixed $p(z)=a_{2 m} z^{2 m}+a_{2 m-2} z^{2 m-2}+\cdots+a_{2} z^{2}$ where $a_{i} \geqslant 0 \forall i, a_{2 m}>0$, the equation

$$
w^{\prime \prime}(z)+(\lambda-p(z)) w(z)=0, \quad \lambda \in \mathbf{R}, z \in \mathbf{C},
$$

has a complete orthonormal set of solutions $\left\{\psi_{n}(z)\right\}_{n=0}^{\infty}$ (eigenfunctions) for $L^{2}(-\infty, \infty)$ (as a real space), with corresponding discrete positive eigenvalues $\left\{\lambda_{n}\right\}_{n=0}^{\infty} . \psi_{n}$ is even or odd as $n$ is even or odd [9, p. 172], and any $L^{2}$ solution of (1) is a multiple of one of these eigenfunctions. [9] has an extensive theory on these matters.

Equation (1) with $p(z)=z^{2}$, yields

$$
\psi_{n}(z)=K_{n} H_{n}(z) \exp \left(-z^{2} / 2\right) \quad\left(\lambda_{n}=2 n+1\right)
$$

where $H_{n}$ is the Hermite polynomial of degree $n$. The well-known recurrence formulas for Hermite polynomials allow the expression of any $g(z) \psi_{N}^{(q)}(z)(g$ a polynomial; fixed $q, N \in \mathbf{N})$ as a finite linear combination of $\left\{\psi_{n}\right\}_{n=0}^{\infty}$. This is shown briefly. The purpose of this paper is to show that when $\operatorname{deg}(p)=2 m$ is a multiple of 4 , any such $g \psi_{N}^{(q)}(g \not \equiv 0$ and $\operatorname{deg}(g) \geqslant 1$ when $q=0)$ cannot be expressed similarly. J. Zeitlin [11] proved this for the special case deg $(p)$ $=4$, by Lie algebra calculations. The crux of the argument here is imaginary

Presented to the Society, January 22, 1976 under the title No recurrence relations for eigenfunctions of certain anharmonic oscillators; received by the editors July 10, 1975.

AMS (MOS) subject classifications (1970). Primary 34A20, 34B25, 42A52, 47E05; Secondary 17B20, 17B30, 17B35, 33A65, 34E20, 42A64.

Key words and phrases. Eigenfunction, eigenvalue, Hermite functions, Hermite polynomials, recurrence relation, complete orthonormal set, anharmonic oscillator, harmonic oscillator, nilpotent Lie algebra, WKB transformation.

${ }^{1}$ This paper is part of the author's doctoral dissertation performed at Rutgers University under the direction of Professor Roe Goodman.

(1) American Mathematical Society 1976 
axis behavior: $\psi_{n}(i y) \rightarrow 0$ but $\psi_{n}^{\prime}(i y) \nrightarrow 0$ as $|y| \rightarrow \infty$ (all $\left.n\right)$. This will be discussed in more detail later.

Remaining as an open question is what happens in the cases $m \geqslant 3$ odd. It would seem that, like here, the answers are negative. A discussion of this is in [5, pp. 31-32].

Our result is of the following interest. In many cases where the eigenfunctions of Sturm-Liouville operators are "known" special functions, there is an associated Lie algebra $\mathcal{G}$ of differential operators $([A, B]=A B-B A)$ whose action on the eigenfunctions yields expressions for $\psi_{N}^{(q)}$, recurrence relations, and other things. For the operator $H=(d / d z)^{2}-p(z), \mathcal{S}$ is the $(m+1)$-step nilpotent Lie algebra with basis $\left\{d / d z, i 1, i z, i z^{2}, \ldots, i z^{m}\right\}$, and our result shows that, for $m$ even, it cannot give finite recurrence relations. It certainly answers R. Goodman's particular question $[10,4.4 .7]$ of whether for fixed $N: \mathcal{G} \cdot \psi_{N}$ $\subset$ linear $\operatorname{span}\left\{\psi_{n}\right\}_{n=0}^{\infty}$ ?

Generalizations can readily be made. Consider the Lie algebraic fact that if $T$ is any differential operator with polynomial coefficients, then $T \psi_{N}$ is expressible as a finite sum of eigenfunctions $\Leftrightarrow[H, T] \psi_{N}$ is also expressible. It follows that, if $T=g(d / d z)^{q}$ as above and $\operatorname{ad} H(T)=[H, T]$, then $(\operatorname{ad} H)^{n}$ - $(T)$ has the nonrecursive property for all $n \in \mathbf{N}$. Furthermore, the proof used on $T$ will generalize to show that the linear span of $\left\{g(d / d z)^{q} \neq\right.$ constant: fixed polynomial $g, q \in \mathbf{N}$ \} also has the nonrecursive property. Obviously, for fixed $q$ and varying $g$, the resulting linear space has the property.

Explaining the title of the paper, $(d / d x)^{2}-q(x)$ is an anharmonic oscillator when $q$ is a polynomial of even degree $\geqslant 4$, with positive leading coefficient. Anharmonic refers to the fact that the eigenvalues are not in an arithmetic progression. There has been intensive recent study of these operators for applications to quantum field theory. See, for example, [2], [3], [8]. Other recent work on these operators is in [6], [7].

II. The eigenfunctions when $p(z)=z^{2}$. In this case, $\psi_{n}(z)=K_{n} H_{n}(z)$ - $\exp \left(-z^{2} / 2\right)$ where $H_{n}$ is the Hermite polynomial of degree $n$. We have the following recurrence relations for Hermite polynomials [1, p. 162]:

LEMMA 1. (i) $H_{n}^{\prime}(z)=2 n H_{n-1}(z)$ for $n \geqslant 1 ; H_{0}^{\prime}(z)=0$.

(ii) $H_{n+1}(z)=2 z H_{n}(z)-2 n H_{n-1}(z)$ for $n \geqslant 1 ; H_{1}(z)=2 z H_{0}(z)$.

Proposition 2. $\forall N, q \in \mathbf{N}$ and polynomial $g: g \psi_{N}^{(q)}$ is always a finite linear combination of $\left\{\psi_{n}\right\}_{n=0}^{\infty}$.

Proof. From Lemma 1, we get

$$
\begin{aligned}
\psi_{N}^{\prime}(z) & =B_{N} \psi_{N-1}(z)+C_{N} \psi_{N+1}(z), & & N \geqslant 1, \\
& =D_{N} \psi_{1}(z), & & N=0 . \\
z \psi_{N}(z) & =b_{N} \psi_{N+1}(z)+c_{N} \psi_{N-1}(z), & & N \geqslant 1, \\
& =d_{N} \psi_{1}(z), & & N=0 .
\end{aligned}
$$


So $\forall k, q \in \mathbf{N}, \psi_{N}^{(q)}(z)$ and $z^{k} \psi_{N}(z)$ are finite linear combinations of $\left\{\psi_{n}\right\}_{n=0}^{\infty}$, and the assertion follows.

Remark. For another proof of Proposition 2, see [5, p. 29].

III. The eigenfunctions when $m$ is even. In this case, on the imaginary axis: $\psi_{N}(i y) \rightarrow 0$ as $|y| \rightarrow \infty \forall$ fixed $N$ (throughout this section, $N$ will be fixed). The argument, the details of which can be found in [5], will now be outlined.

Define $\phi: \mathbf{R} \rightarrow \mathbf{R}$ by

$$
\begin{aligned}
\phi(y) & =\psi_{N}(i y) & & \text { if } N \text { is even } \\
& =i \psi_{N}(i y) & & \text { if } N \text { is odd. }
\end{aligned}
$$

Then equation (1) gives

$$
\phi^{\prime \prime}(y)+\left(p(i y)-\lambda_{N}\right) \phi(y)=0 .
$$

Pick $y_{0}$ so that $p(i y)>\lambda_{N}$ for $y \geqslant y_{0}$. Then for $y \geqslant y_{0}$, the transformations (WKB)

$$
\xi(y)=\int_{y_{0}}^{y}\left(p(i u)-\lambda_{N}\right)^{1 / 2} d u, \quad \eta(y)+\left(p(i y)-\lambda_{N}\right)^{1 / 4} \phi(y)
$$

take equation (3) into

$$
\frac{d^{2} \eta}{d \xi^{2}}+\eta(y)+\eta(y)\left[\frac{p^{\prime \prime}(i y)}{4\left(p(i y)-\lambda_{N}\right)^{2}}-\frac{5\left(p^{\prime}(i y)\right)^{2}}{16\left(p(i y)-\lambda_{N}\right)^{3}}\right]=0 .
$$

Note that the last coefficient of $\eta$ goes to zero as $|y| \rightarrow \infty$. All solutions $\eta=\theta(\xi)$ of (5) satisfy the integral equation

$$
\theta(\xi)=\theta(0) \cos \xi+\theta^{\prime}(0) \sin \xi-\int_{0}^{\xi} \sin (\xi-T) R(t) \theta(T) d T
$$

where

$$
T=\xi(t) \quad \text { and } \quad R(t)=\frac{p^{\prime \prime}(i t)}{4\left(p(i t)-\lambda_{N}\right)^{2}}-\frac{5\left(p^{\prime}(i t)\right)^{2}}{16\left(p(i t)-\lambda_{N}\right)^{3}} .
$$

From this integral equation it can be deduced that: for $A, B$ constants (not both zero),

(6) $\phi(y)=\left(p(i y)-\lambda_{N}\right)^{-1 / 4}[A \cos \xi(y)+B \sin \xi(y)+o(1)]$ as $y \rightarrow \infty$.

This method of deriving (6) from a general equation, similar to (3), can be found in 5.8 and 5.10 of [9].

(6) shows, among other things, that $\psi_{N} \rightarrow 0$ along the imaginary axis (by (2)). Curiously, $\psi_{N}^{\prime} \nrightarrow 0$ along the imaginary axis. Although this fact comes out in the course of obtaining (6) [5, p. 27], below we give an elementary proof which uses none of the machinery developed to get to (6).

Because $p(i y) \rightarrow \infty$ as $|y| \rightarrow \infty$, (2) and (3) imply that $\psi_{N}$ has an infinite 
number of pure imaginary zeros (for $y \geqslant y_{0}$, compare (3) with $f^{\prime \prime}(y)+f(y)$ $=0$ and use Sturm's theorem on p. 107 of [9]). This oscillatory character makes (6) believable.

Definition 3. Let $\left\{i A_{\nu}\right\}_{\nu=1}^{\infty}$ be the pure imaginary zeros of $\psi_{N}$ with $A_{\nu}>0$ (ordered by increasing modulus).

Lemma 4. Choose $A>0$ so that $i p^{\prime}(i y)>0$ for $y \geqslant A$. Then for $A_{\nu}>A$; that is, for $\nu \geqslant \nu_{0}$ :

$$
\left|\psi_{N}{ }^{\prime}\left(i A_{\nu}\right)\right|^{2}>\left(p(i A)-\lambda_{N}\right)\left|\psi_{N}(i A)\right|^{2}+\left|\psi_{N}{ }^{\prime}(i A)\right|^{2} .
$$

Proof. For $\nu \geqslant \nu_{0}$, integration by parts gives

$$
\begin{gathered}
\int_{A}^{A_{\nu}} \phi^{\prime \prime}(y) \phi^{\prime}(y) d y=\int_{A}^{A_{\nu}}\left(\lambda_{N}-p(i y)\right) \phi(y) \phi^{\prime}(y) d y, \\
\left(\phi^{\prime}\left(A_{\nu}\right)\right)^{2}-\left(\phi^{\prime}(A)\right)^{2}=(\phi(A))^{2}\left(p(i A)-\lambda_{N}\right)+\int_{A}^{A_{\nu}} i p^{\prime}(i y)(\phi(y))^{2} d y \\
>(\phi(A))^{2}\left(p(i A)-\lambda_{N}\right)
\end{gathered}
$$

and the result follows from (2).

Corollary 5. $\left|\psi_{N}^{\prime}\left(i A_{\nu}\right)\right|>\left|\psi_{N}^{\prime}\left(i A_{\nu-1}\right)\right| \forall \nu \geqslant \nu_{0}+1$.

Proof. Set $A=A_{\nu-1}$ in Lemma 4.

THeOREM 6. $\forall q, N \in \mathbf{N}$ and polynomial $g \not \equiv 0(\operatorname{deg}(g) \geqslant 1$ when $q=0)$ : $g \psi_{N}^{(g)}$ cannot be expressed as a finite linear combination of $\left\{\psi_{n}\right\}_{n=0}^{\infty}$.

Proof. Assume the contrary. Then an induction argument on the derivatives of $\psi_{N}^{(2)}(z)=\left(p(z)-\lambda_{N}\right) \psi_{N}(z)$ gives $\forall q \geqslant 1(q \neq 2)$ :

$$
g(z) \psi_{N}^{(q)}(z)=g_{1}(z) \psi_{N}(z)+g_{2}(z) \psi_{N}^{\prime}(z)=\sum_{k=0}^{K} c_{k} \psi_{n_{k}}(z), \quad z \in \mathbf{C},
$$

where $g_{1}, g_{2}$ are polynomials with $g_{2} \not \equiv 0$. Therefore

$$
\lim _{\nu \rightarrow \infty} g_{2}\left(i A_{\nu}\right) \psi_{N}^{\prime}\left(i A_{\nu}\right)=\lim _{\nu \rightarrow \infty} \sum_{k=0}^{K} c_{k} \psi_{n_{k}}\left(i A_{\nu}\right)
$$

Since every eigenfunction goes to zero along the imaginary axis ((6) with (2)) $\Rightarrow$ the right side above is zero. But by Corollary 5 , the left side is not zero; hence a contradiction.

Now suppose $g(z) \psi_{N}(z)=\sum_{k=0}^{K} c_{k} \psi_{n_{k}}(z)$ where deg $(g) \geqslant 1$. Differentiating twice:

$$
\begin{gathered}
g^{\prime \prime}(z) \psi_{N}(z)+2 g^{\prime}(z) \psi_{N}^{\prime}(z)+\left(g(z) \psi_{N}(z)\right)\left(p(z)-\lambda_{N}\right) \\
=\sum_{k=0}^{K} c_{k}\left(p(z)-\lambda_{n_{k}}\right) \psi_{n_{k}}(z) \\
g^{\prime \prime}(z) \psi_{N}(z)+2 g^{\prime}(z) \psi_{N}^{\prime}(z)=\sum_{k=0}^{K} c_{k}\left(\lambda_{N}-\lambda_{n_{k}}\right) \psi_{n_{k}}(z)
\end{gathered}
$$


Again we get a contradiction by the previous argument.

Finally, if $g(z) \psi_{N}^{(2)}(z)=g(z)\left(p(z)-\lambda_{N}\right) \psi_{N}(z)=\sum_{k=0}^{K} c_{k} \psi_{n_{k}}(z)$, this would contradict what was just proven.

The proof is now complete.

\section{REFERENCES}

1. W. W. Bell, Special functions for scientists and engineers, Van Nostrand, Princeton, N.J., 1968. MR 46 \#2087a.

2. C. M. Bender and T. T. Wu, Phys. Rev. Letters 21 (1968), 406.

3. - Anharmonic oscillator, Phys. Rev. (2) 184 (1969), 1231-1260. MR 41 \#4951.

4. I. M. Gel'fand and G. E. Shilov, Generalized functions. Vol. 2, Spaces of fundamental and generalized functions, Fizmatgiz, Moscow, 1958; English transl., Academic Press; Gordon and Breach, New York, 1968. MR 21 \#5142a; 37 \#693.

5. G. G. Gundersen, Eigenfunctions and Cauchy problems for anharmonic and harmonic oscillators, Thesis, Rutgers University, 1975.

6. P. F. Hsieh and Y. Sibuya, On the asymptotic integration of second order linear ordinary differential equations with polynomial coefficients, J. Math. Anal. Appl. 16 (1966), 84-103. MR 34 \#403.

7. J. S. Rosen, Logarithmic Sobolev inequalities and supercontractivity for anharmonic oscillators, Thesis, Princeton University, 1974.

8. B. Simon, Pointwise bounds on eigenfunctions and wave packets in $N$-body quantum systems. III, Trans. Amer. Math. Soc. 208 (1975), 317-329.

9. E. C. Titchmarsh, Eigenfunction expansions associated with second-order differential equations. Part I, 2nd ed. Clarenden Press, Oxford, 1962. MR 8, 458; 31 \# 426.

10. G. Warner, Harmonic analysis on semi-simple Lie groups I, Springer-Verlag, New York, 1972.

11. J. Zeitlin, Correspondence between Lie algebra invariant subspaces and Lie group invariant subspaces of representations of Lie groups, Trans. Amer. Math. Soc. 167 (1972), 227-242. MR 45 \#6980.

Department of Mathematics, University of New Orleans, New Orleans, louisiana 70122 